\title{
Early hyperoxemia may not increase mortality after cardiac arrest: a pilot study
}

\section{Young Taeck $\mathrm{Oh}^{1}$,Yong Hwan Kim², You Dong Sohn ${ }^{1}$, Seung Min Park', Dong Hyuk Shin ${ }^{3}$, Seong Youn Hwang ${ }^{2}$, Suck Ju Cho ${ }^{4}$, Sang 0 Park ${ }^{5}$, Chong Kun Hong ${ }^{6}$, Hee Cheol Ahn', Young Hwan Lee ${ }^{1}$}

\author{
'Department of Emergency Medicine, Hallym University Sacred Heart Hospital, Hallym University School of \\ Medicine, Anyang, Korea \\ ${ }^{2}$ Department of Emergency Medicine, Samsung Changwon Hospital, Sungkyunkwan University School of \\ Medicine, Changwon, Korea \\ ${ }^{3}$ Department of Emergency Medicine, Kangbuk Samsung Hospital, Sungkyunkwan University School of \\ Medicine, Seoul, Korea \\ ${ }^{4}$ Department of Emergency Medicine, Pusan National University Hospital, Busan, Korea \\ ${ }^{5}$ Department of Emergency Medicine, Konkuk University Medical Center, Seoul, Korea \\ ${ }^{6}$ Department of Emergency Medicine, Bundang Jesaeng General Hospital, Seongnam, Korea
}

Objective International Liaison Committee on Resuscitation guidelines advocate an arterial saturation of 94\% to 96\% after return of spontaneous circulation (ROSC). However, a few clinical trials have investigated the impact of postresuscitative $\mathrm{O}_{2}$ therapy after cardiac arrest. We studied whether early hyperoxemia is associated with a poor post-ROSC outcome after in-hospital cardiac arrest.

Methods We retrospectively reviewed patients who experienced an in-hospital cardiac arrest from January 2005 to January 2011. Based on the results of the first arterial blood gas analysis (ABGA) within 10 minutes and a second ABGA from 60 to 120 minutes after ROSC, patients were classified into three groups: hyperoxemia $\left(\mathrm{PaO}_{2} \geq 300 \mathrm{mmHg}\right)$, normoxemia $(300 \mathrm{mmHg}>$ $\left.\mathrm{PaO}_{2} \geq 60 \mathrm{mmHg}\right)$, and hypoxemia $\left(\mathrm{PaO}_{2}<60 \mathrm{mmHg}\right.$ or ratio of $\mathrm{PaO}_{2}$ to fraction of inspired oxygen $<300$ ). We examined whether early hyperoxemia was associated with survival and neurological outcome.

Results There were 792 patients who met the inclusion criteria: 638 (80.6\%) in the hypoxemia group, $62(7.8 \%)$ in the normoxemia group, and $92(11.6 \%)$ in the hyperoxemia group. Multiple logistic regression analysis showed that hyperoxemia was not associated with survival (odds ratio $[\mathrm{OR}], 1.07 ; 95 \%$ confidence interval $[\mathrm{Cl}], 0.30$ to 3.84$)$ or neurological outcome $(\mathrm{OR}, 1.03$; $95 \% \mathrm{Cl}, 0.31$ to 3.40$)$.

Conclusion Postresuscitation hyperoxemia was not associated with survival or neurological outcome in patients with ROSC after in-hospital cardiac arrest.

Keywords Hyperoxemia; Cardiopulmonary resuscitation; Mortality
elSSN: 2383-4625

Received: 3 July 2014

Revised: 16 August 2014

Accepted: 18 August 2014

Correspondence to: Young Hwan Lee Department of Emergency Medicine, Hallym University Sacred Heart Hospital, Hallym University School of Medicine, 22 Gwanpyeong-ro 170beon-gil, Dongan-gu, Anyang 431-796, Korea

E-mail: hwaniyo@naver.com

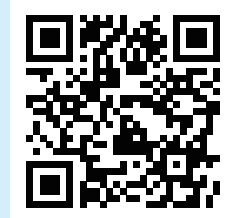

How to cite this article:

Oh YT, Kim YH, Sohn YD, Park SM, Shin DH, Hwang SY, Cho SJ, Park SO, Hong CK, Ahn $\mathrm{HC}$, Lee YH. Early hyperoxemia may not increase mortality after cardiac arrest: a pilot study. Clin Exp Emerg Med 2014;1(1): 28-34.

This is an Open Access article distributed under the terms of the Creative Commons Attribution Non-Commercial License (http:// creativecommons.org/licenses/by-nc/3.0/). 


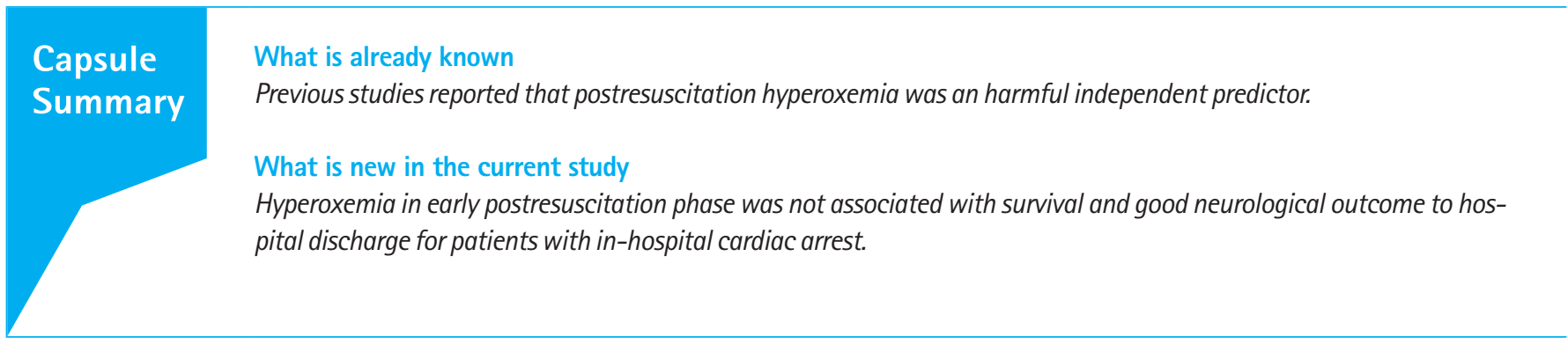

\section{INTRODUCTION}

Current American Heart Association (AHA) guidelines for adult cardiopulmonary resuscitation (CPR) recommend 100\% inspired oxygen during resuscitative efforts to maximize the likelihood of achieving return of spontaneous circulation (ROSC). After ROSC is achieved, the International Liaison Committee on Resuscitation guidelines advocate a controlled reoxygenation strategy targeting an arterial oxygen saturation of 94\% to 96\%., Experimental evidence suggests that routine administration of high concentrations of $\mathrm{O}_{2}$ can contribute to significant neurological injury after ROSC and that even short periods of postresuscitative hyperoxemia may cause oxidative neuronal injury and worsen the neurological outcome. $^{3-8}$

Several clinical trials have investigated postresuscitative $\mathrm{O}_{2}$ therapy after cardiac arrest. In two large retrospective studies of adult patients admitted to the intensive care unit (ICU) after resuscitation from cardiac arrest, Kilgannon et al. ${ }^{9,10}$ reported that postresuscitation hyperoxemia was an independent predictor of mortality and poor neurological outcome, and that the relationship was dose-dependent. However, in their study, the measured variables were based only on the first $\mathrm{PaO}_{2}$ value at an unspecified time within 24 hours of admission. Several important variables known to influence survival after CPR, such as CPR duration and initial rhythm, were not adjusted for in their study. ${ }^{11-14}$ In contrast, observational data suggest that hyperoxemia has no robust or consistently reproducible relationship with mortality. ${ }^{15}$ One randomized prospective clinical trial compared the effect of ventilation for the first 60 minutes with a fraction of inspired oxygen $\left(\mathrm{FiO}_{2}\right)$ of 0.3 and 1.0 and found no significant difference in outcomes at hospital discharge after ROSC following out-of-hospital cardiac arrest. ${ }^{16}$ Some experimental data had shown that postischemic hyperbaric oxygen can afford neuroprotection after experimental cardiac arrest-induced complete global ischemia. ${ }^{17-19}$

Oxygen plays complex biological roles, and it is difficult to define the effects on outcome after both hypoxemia and hyperoxemia. This study was implemented to test the hypothesis that early exposure to hyperoxemia is adversely associated with the clinical outcome after ROSC from in-hospital cardiac arrest.

\section{METHODS}

This was a retrospective observational study of patients with ROSC after in-hospital cardiac arrest in our cardiac arrest database, which contains data from two hospitals from January 2005 to January 2011.

The CPR team of our hospital comprises an emergency physician, nurses, and paramedics who are certified as AHA-accredited advanced cardiac life-support providers. They respond to most cases of in-hospital cardiac arrest, except for cases in the ICU, which are treated by intensivists within the ICU. Patients were excluded if they were younger than 17 years or had traumatic cardiac arrest or were under percutaneous cardio-pulmonary support (PCPS) at the moment of cardiac arrest. We retrospectively reviewed the medical records of adult patients with ROSC after in-hospital non-traumatic cardiac arrest.

This study was approved by the Institutional Review Board of Hallym University Sacred Heart Hospital. We retrospectively analyzed a historical data set that was drawn from the in-hospital CPR registry between 2005 and 2011 according to Utstein style guidelines. ${ }^{20}$ Additional data were collected from electronic medical records: socio-demographics, comorbidities, cause of cardiac arrest, CPR duration, place of cardiac arrest, initial monitored rhythm, survival to hospital discharge (alive or dead), and neurological status at hospital discharge.. ${ }^{21}$ The severity of organ dysfunction was measured using the Sequential Organ Failure Assessment (SOFA) before the cardiac arrest.

We categorized oxygenation levels into the same three groups as in the EMShockNet study. ${ }^{10}$ Patients were classified into three groups based on the results of the first (within 10 minutes of ROSC) arterial blood gas analysis (ABGA) and the second ABGA from 60 minutes to 120 minutes after ROSC. Hyperoxemia was defined as $\mathrm{PaO}_{2}$ of $\geq 300 \mathrm{mmHg}_{1}^{22}$ hypoxemia as $\mathrm{PaO}_{2}$ of $<60$ $\mathrm{mmHg}$ (or ratio of $\mathrm{PaO}_{2}$ to $\mathrm{FIO}_{2}<300$ ) ${ }_{i}^{23}$ and normoxemia, as cases not classified as hyperoxemia or hypoxemia.

Cases where the results of the first and second ABGA were 
classified into different groups were excluded from the study.

The primary outcome of the current study was survival to hospital discharge. The secondary outcome measure was a "good outcome" defined as discharge to home or an acute rehabilitation facility. Good outcome was defined as a summation of Cerebral Performance Category (CPC) of 1 or 2 with survival to hospital discharge.

Continuous variables are expressed as the mean \pm standard deviation (SD) and median with interquartile range (IOR). Categorical data are reported as percentage and 95\% confidence interval (CI). The Wilcoxon rank-sum test or the Kruskal-Wallis test was used to compare continuous variables, and the chi-square test or Fisher exact test was used to compare categorical variables. The occurrence of the primary outcome was compared between the groups using the chi-square test. We also performed univariate analysis using the chi-square test or Fisher exact test for nominal variables, the t-test for means, and the Mann-Whitney U-test for medians to assess the correlations between survival and several variables and between good neurological outcome and several variables. Hyperoxemia and other factors that were significantly associated with survival to discharge and good neurological outcome in the univariate analysis were analyzed by multivariable logistic regression analysis. P-value $<0.05$ was considered significant. We used the SPSS ver. 18 (SPSS Inc., Chicago, IL, USA) for all statistical analyses.

\section{RESULTS}

There were 2,644 patients who had in-hospital cardiac arrest. Of

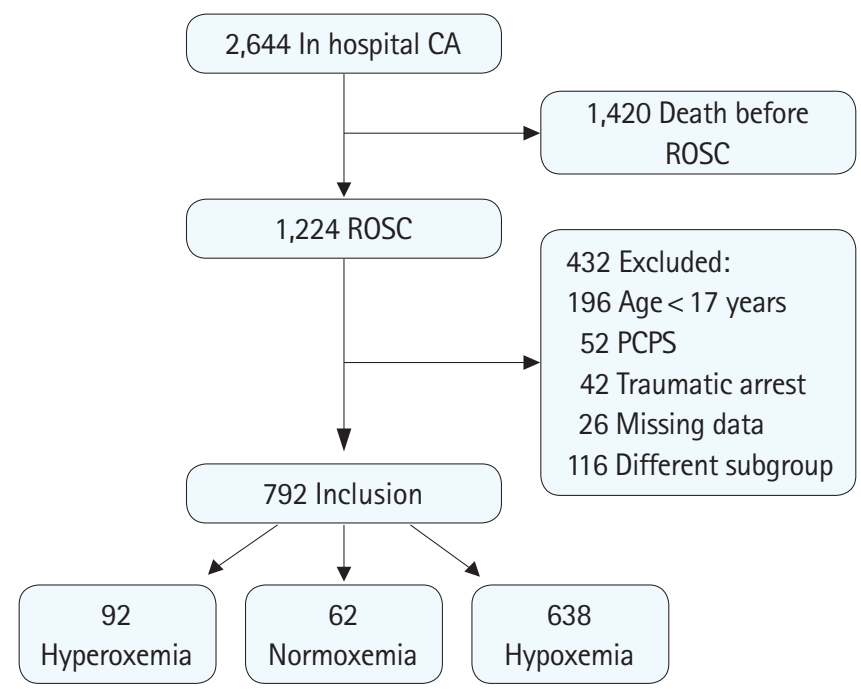

Fig. 1. In-hospital cardiac arrest patients between January 2005 and January 2011. CA, cardiac arrest; ROSC, return to spontaneous circulation; PCPS, percutaneous cardiopulmonary support. these, 1,852 patients $(70 \%)$ were excluded: $1,420(53.7 \%)$ had no ROSC, 196 (7.4\%) were younger than 17 years, 52 (3.9\%) were supported by PCPS, 116 (2.4\%) belonged to different groups in the first and second ABGA results, 42 (1.6\%) experienced traumatic arrest, and 26 (1.0\%) had missing arterial blood gas data (Fig. 1). ROSC was achieved in 1,224 cases (46.3\%). The number of surviving patients at discharge was $272(10.3 \%)$, and the number of patients who had good neurological outcome was 196 (7.4\%) of in-hospital cardiac arrest.

Table 1. Demographic characteristics of the study patients

\begin{tabular}{lcccc}
\hline & & \multicolumn{3}{c}{ First and second ABGA PaO } \\
\cline { 3 - 5 } Variable & $\begin{array}{c}\text { Total } \\
(n=792)\end{array}$ & $\begin{array}{c}\text { Hyperoxemia } \\
(n=92)\end{array}$ & $\begin{array}{c}\text { Normoxemia } \\
(n=62)\end{array}$ & $\begin{array}{c}\text { Hypoxemia } \\
(n=638)\end{array}$ \\
\hline Age (yr), mean \pm SD & $63.6 \pm 11.5$ & $64.4 \pm 10.4$ & $67.1 \pm 9.1$ & $62.6 \pm 10.5$ \\
Male & $512(64.6)$ & $55(60.0)$ & $26(41.9)^{\mathrm{a})}$ & $411(64.4)$ \\
Comorbidities & & & & \\
$\quad$ Diabetes mellitus & $266(33.6)$ & $28(30.4)$ & $20(32.3)$ & $218(34.1)$ \\
Hypertension & $286(36.1)$ & $28(30.4)$ & $22(35.5)$ & $236(37.0)$ \\
Chronic renal disease & $120(15.2)$ & $14(15.2)$ & $10(16.1)$ & $96(15.0)$ \\
Chronic heart disease & $316(39.9)$ & $46(50.0)$ & $30(48.4)$ & $240(37.6)$ \\
Chronic hepatic disease & $126(15.9)$ & $16(17.4)$ & $6(9.7)$ & $104(16.3)$ \\
Malignancy & $254(32.1)$ & $26(28.3)$ & $14(22.6)$ & $214(33.5)$ \\
Respiratory insufficiency & $74(9.4)$ & $10(10.9)$ & $4(6.5)$ & $60(9.4)$ \\
Stroke & $82(10.4)$ & $8(8.7)$ & $8(12.9)$ & $66(10.3)$ \\
SOFA score, median (IQR) & $5.0(3.8-10.0)$ & $5.0(3.0-8.3)$ & $5.0(2.0-9.0)^{b)}$ & $7.0(4.0-10.0)$ \\
\hline
\end{tabular}

Values are presented as number (\%) unless otherwise indicated. ABGA, arterial blood gas analysis; SOFA, Sequential Organ Failure Assessment. ${ }^{a} \mathrm{P}=0.02$ for comparison of normoxemia with hypoxemia. ${ }^{\text {b) }} \mathrm{P}=0.04$ for comparison of normoxemia with hypoxemia.

Table 2. Descriptive data of cardiac arrest

\begin{tabular}{|c|c|c|c|c|}
\hline \multirow[b]{2}{*}{ Variable } & \multirow{2}{*}{$\begin{array}{c}\text { Total } \\
(n=792)\end{array}$} & \multicolumn{3}{|c|}{ First and second $\mathrm{ABGA} \mathrm{PaO}_{2}$} \\
\hline & & $\begin{array}{c}\text { Hyperoxemia } \\
(n=92)\end{array}$ & $\begin{array}{l}\text { Normoxemia } \\
\qquad(n=62)\end{array}$ & $\begin{array}{c}\text { Hypoxemia } \\
(\mathrm{n}=638)\end{array}$ \\
\hline $\begin{array}{l}\text { Initial shockable } \\
\text { rhythm }\end{array}$ & $156(20.8)$ & $20(25)$ & $18(31)$ & $118(19.3)$ \\
\hline \multicolumn{5}{|l|}{ Place of cardiac arrest } \\
\hline Intensive care unit & $228(28.8)$ & $24(26.1)$ & $30(48.4)^{a)}$ & $174(27.3)$ \\
\hline Ward & $342(43.2)$ & $34(37.0)$ & $10(16.1)^{\mathrm{a})}$ & $298(46.7)$ \\
\hline Other & $222(28.0)$ & $34(37.0)$ & $22(35.5)$ & $166(26.0)$ \\
\hline CPR duration (min) & $14.2 \pm 14.7$ & $16.3 \pm 15.0$ & $11.2 \pm 12.1$ & $14.1 \pm 14.9$ \\
\hline \multicolumn{5}{|l|}{ Cause of arrest } \\
\hline Cardiac & $236(29.8)$ & $30(32.6)$ & $24(38.7)$ & $182(28.5)$ \\
\hline Respiratory & $172(21.7)$ & $26(28.3)$ & $4(6.5)$ & $142(22.3)$ \\
\hline Other & $384(48.5)$ & $36(39.1)$ & $34(54.8)$ & $314(49.2)$ \\
\hline $\begin{array}{l}\text { Time between the } \\
\text { 1st and 2nd ABGA } \\
\text { (min) }\end{array}$ & $\begin{array}{c}70 \\
(64-86.2)\end{array}$ & $\begin{array}{c}74.5 \\
(65.1-88.3)\end{array}$ & $\begin{array}{c}71 \\
(68.5-98.5)\end{array}$ & $\begin{array}{c}63 \\
(61-70.2)\end{array}$ \\
\hline
\end{tabular}

Values are presented as number $(\%)$, mean $\pm S D$, or median (interquartile range). $A B G A$, arterial blood gas analysis; CPR, cardiopulmonary resuscitation; IQR, interquartile range.

${ }^{\text {a) }} \mathrm{P}<0.001$ for comparison of normoxemia with hypoxemia. 
Table 3. Outcomes of the study patients

\begin{tabular}{lccccc}
\hline \multirow{2}{*}{ Outcome } & \multirow{2}{*}{$\begin{array}{c}\text { Total } \\
(n=792)\end{array}$} & Hyperoxemia $(n=92)$ & Normoxemia $(n=62)$ & Hypoxemia $(n=638)$ & First and second $\mathrm{PaO}_{2}$ \\
\cline { 3 - 6 } & $272(34.3)$ & $34(37.0)$ & $30(48.4)$ & $208(32.6)$ & 0.19 \\
Survival discharge & $196(24.7)$ & $24(26.1)$ & $22(35.5)$ & $150(23.5)$ & 0.33
\end{tabular}

Values are presented as number (\%).

Table 4. Multivariable logistic regression and odds ratios of survival to hospital discharge

\begin{tabular}{lccc}
\hline Variable & Odds ratio & $95 \% \mathrm{Cl}$ & P-value \\
\hline Age & 0.96 & $0.94-0.99$ & 0.002 \\
$\begin{array}{l}\text { Sequential organ failure } \\
\text { assessment }\end{array}$ & 0.59 & $0.19-0.79$ & 0.01 \\
$\begin{array}{l}\text { Cause of arrest } \\
\quad \text { Cardiac origin }\end{array}$ & & & \\
$\quad$ Respiratory origin & 0.33 & $0.42-2.57$ & 0.44 \\
CPR duration & 0.89 & $0.25-1.41$ & 0.96 \\
Shockable rhythm & 1.94 & $1.43-2.14$ & $<0.001$ \\
First and second ABGA PaO 2 & & & \\
$\quad$ Normoxia & & & \\
$\quad$ Hyperoxemia & 1.00 (reference) & & \\
$\quad$ Hypoxemia & 1.03 & $0.31-3.40$ & 0.96 \\
& 0.70 & $0.28-1.73$ & 0.44
\end{tabular}

$\mathrm{Cl}$, confidence interval; $\mathrm{CPR}$, cardiopulmonary resuscitation; $\mathrm{ABGA}$, arterial blood gas analysis.

Seven hundred and ninety-two adults were included in this study. The mean age of the patients was $63.6 \pm 11.5$ years, and $64.6 \%$ were men. Six hundred and thirty-eight patients (80.6\%) were classified into the hypoxemia group, $92(11.6 \%)$ into the hyperoxemia group, and $62(7.8 \%)$ into the normoxemia group. The baseline characteristics are presented in Tables 1, 2. The three groups differed significantly in sex distribution, SOFA score, and place of cardiac arrest. Patients with normoxia had less severe illness as reflected in lower baseline SOFA scores than the hypoxemia group $(P<0.05)$. Normoxemia was more frequent in patients who experienced cardiac arrest in the ICU $(P<0.001)$, and hypoxemia was more frequent in those who experienced cardiac arrest in the ward $(\mathrm{P}<0.001)$.

After ROSC, $34.3 \%$ of the patients survived to discharge, and $24.7 \%$ had good neurological outcome. Hyperoxemia was not significantly associated with survival to discharge $(P=0.19)$ or with good neurological outcome at the time of discharge $(\mathrm{P}=0.33)$ (Table 3).

In univariate analysis, age, SOFA score, cause of arrest, CPR duration, and shockable rhythm were significantly associated with survival to discharge and good neurological outcome. $\mathrm{PaO}_{2}$, age, SOFA score, cause of arrest, CPR duration, and shockable rhythm were included in the multivariable logistic regression analysis. Early exposure to hyperoxemia was not significantly associated with survival to discharge and good neurological out-
Table 5. Multivariable logistic regression and odds ratios of good neurological outcome at hospital discharge

\begin{tabular}{lccc}
\hline Variable & Odds ratio & 95\% Cl & P-value \\
\hline Age & 0.95 & $0.92-0.98$ & $<0.001$ \\
$\begin{array}{l}\text { Sequential organ failure } \\
\quad \text { assessment }\end{array}$ & 0.52 & $0.16-0.78$ & 0.07 \\
$\begin{array}{l}\text { Cause of arrest } \\
\quad \text { Cardiac origin }\end{array}$ & 1.55 & $0.54-4.47$ & 0.42 \\
$\quad$ Respiratory origin & 0.66 & $0.55-1.02$ & 0.37 \\
CPR duration & 0.88 & $0.84-0.93$ & $<0.001$ \\
Shockable rhythm & 1.63 & $1.42-2.26$ & 0.002 \\
First and second ABGA PaO 2 & & & \\
$\quad$ Normoxia & & & \\
$\quad$ Hyperoxemia & 1.00 (reference) & & \\
$\quad$ Hypoxemia & 1.07 & $0.30-3.84$ & 0.91 \\
\hline
\end{tabular}

$\mathrm{Cl}$, confidence interval; $\mathrm{CPR}$, cardiopulmonary resuscitation; $\mathrm{ABGA}$, arterial blood gas analysis.

come after adjusting for all other significant factors (Tables 4, 5).

Age, CPR duration, shockable rhythm, and SOFA score were significantly associated with survival to discharge and good neurological outcome (Tables 4, 5). The Hosmer-Lemeshow goodnessof-fit P-values were 0.674 and 0.622 , respectively.

\section{DISCUSSION}

In the current study, hyperoxemia was relatively uncommon in the provision of ROSC after in-hospital cardiac arrest, and the $\mathrm{PaO}_{2}$ of the first $A B G A$ within 10 minutes and the second $A B G A$ from 60 to 120 minutes after ROSC were not associated with survival to discharge or good neurological outcome for in-hospital cardiac arrest patients. Hyperoxemia was not significantly associated with survival to discharge or with good neurological outcome in the univariate or multiple regression analyses.

Our results differ from those of recent large multicenter cohort studies of Project IMPACT database adult patients by Kilgannon et al. ${ }^{9,10}$ There are several important differences between these prior studies and our study. First, Kilgannon et al., ${ }^{910}$ analyzed only the first ICU ABGA within 24 hours after ROSC. Although the duration of exposure to hyperoxemia may be critical, the study did not analyze the time. Another large multicenter cohort study also attempted to correlate the lowest blood gas measurement with 
the mean $\mathrm{PaO}_{2}$ in the first 24 hours, and the duration of exposure to hyperoxemia was not identified in the study. ${ }^{15}$ We performed the first ABGA within 10 minutes and the second ABGA from 60 to 120 minutes after ROSC to analyze the effects of hyperoxemia in the early resuscitation phase. Even though continuous $\mathrm{SpO}_{2}$ monitoring and oxygen back-up were not measured during the early resuscitation phase, we included only those patients classified into the same subgroup in the first and second ABGA. The median time did not differ significantly between the $\mathrm{PaO}_{2}$ subgroups, and the median time between the first and second ABGA was 70 minutes (IQR, 64 to 86.2). The time may be the duration of exposure to hyperoxemia in the hyperoxemia group.

Second, the SOFA score, CPR duration, and shockable rhythm were uncontrolled in the previous studies. ${ }^{9,10} \mathrm{CPR}$ duration and rhythm have also been associated with survival to discharge from hospital after ROSC in clinical trials. ${ }^{12,13,24}$ In our study, CPR duration had a wide range (median, 10 minutes; IQR, 5 to 19), and CPR duration and rhythm were strong factors for survival. In our multiple regression analysis, we controlled for known important factors for survival such as CPR duration, shockable rhythm, and SOFA score and found that $\mathrm{PaO}_{2}$ was not a significant predictor. $^{11-14}$

In the initial reperfusion phase, autoregulation of cerebral blood flow is impaired for some time after cardiac arrest, and hypoxemia can compromise cerebral oxygen delivery and possibly cause secondary injury after cardiac arrest. ${ }^{19}$ In neonatal resuscitation, $100 \% \mathrm{O}_{2}$ is deleterious to the brain. ${ }^{25}$ However, because infants have compromised antioxidant defenses and because of age-related differences in endogenous defenses against hypoxemia, the risk of $100 \% \mathrm{O}_{2}$ may be magnified considerably in neonates compared with adults. ${ }^{26}$ Experimental studies on early hyperoxemia after resuscitation from experimental cardiopulmonary arrest have shown deleterious effects. ${ }^{27,28}$ However, we emphasize that the experimental data reflect the effect of hyperoxemic reperfusion on ischemic brain injury in healthy animals. This is very different from the clinical situation, because many patients with cardiac arrest are not young and have multiple comorbidities. In our study, the mean $( \pm S D)$ age was $63.6 \pm 11.5$ years; 316 patients (39.9\%) had chronic heart disease and 254 patients (32.1\%) had malignancy. On the contrary, there were 182 healthy patients (23\%) without any comorbidity. Such chronically ill patients may require a high $\mathrm{FiO}_{2}$ for adequate oxygenation. Several studies reveal that ventilation with $100 \% \mathrm{O}_{2}$ for the first hour after ROSC can produce worse neurological outcomes than immediate adjustment of the $\mathrm{FiO}_{2}$ to produce an $\mathrm{O}_{2}$ arterial saturation of $94 \%$ to $96 \% .^{3,4,6-8,29}$ However, this controlled reoxygenation strategy has yet to be studied in clinical trials.
In our study, after controlling for several factors and classifying patients according to the two ABGAs, early exposure to hyperoxemia after ROSC subsequent to in-hospital cardiac arrest was not associated with survival and good neurological outcome.

However, several limitations of this work should be noted. First, this was an observational study. Further studies involving randomized prospective clinical trials are needed to understand fully the effects of hyperoxemia after ROSC. Second, we excluded patients with PCPS during CPR. In our pilot study, patients with PCPS had a high $\mathrm{O}_{2}$ level and survival compared with those treated with conventional CPR. ${ }^{30,31}$ Because therapeutic hypothermia was not widely applied in this cohort, we did not investigate the role of therapeutic hypothermia, which is recommended in treating cardiac arrest of cardiac origin. ${ }^{30}$ Identifying the additive or synergistic effects of hypothermia and PCPS requires further study. Third, we have focused on the concern about the potential adverse effects of early hyperoxemia after resuscitation from cardiac arrest. However, our study did not have similar patient sets for post-ROSC hyperoxemia or normoxemia for outcomes comparison; this is because our study results were from a two-center experience with a relatively small population. Fourth, data were collected in two hospitals over six years, and the enrolled number of patients may be too small to draw a statistically powerful conclusion. Further multicenter study is necessary to confirm this result. Fifth, we could not confirm the exact duration of hyperoxemia, and those who had different results between the two consecutive ABGAs were arbitrarily excluded. This exclusion may have resulted in a selection bias to some degree. Sixth, we did not perform a six-month follow-up and cannot draw definite conclusions about the long-term outcomes.

In summary, our study showed that exposure to hyperoxemia in the early postresuscitation phase was not associated with better survival and good neurological outcome to hospital discharge for patients with ROSC after in-hospital cardiac arrest.

\section{CONFLICT OF INTEREST}

No potential conflict of interest relevant to this article was reported.

\section{REFERENCES}

1. Neumar RW, Nolan JP, Adrie C, et al. Post-cardiac arrest syndrome: epidemiology, pathophysiology, treatment, and prognostication. A consensus statement from the International Liaison Committee on Resuscitation (American Heart Association, Australian and New Zealand Council on Resuscitation, 
European Resuscitation Council, Heart and Stroke Foundation of Canada, InterAmerican Heart Foundation, Resuscitation Council of Asia, and the Resuscitation Council of Southern Africa); the American Heart Association Emergency Cardiovascular Care Committee; the Council on Cardiovascular Surgery and Anesthesia; the Council on Cardiopulmonary, Perioperative, and Critical Care; the Council on Clinical Cardiology; and the Stroke Council. Circulation 2008;118:2452-83.

2. Peberdy MA, Callaway CW, Neumar RW, et al. Part 9: postcardiac arrest care: 2010 American Heart Association Guidelines for Cardiopulmonary Resuscitation and Emergency Cardiovascular Care. Circulation 2010;122(18 Suppl 3):S768-86.

3. Balan IS, Fiskum G, Hazelton J, Cotto-Cumba C, Rosenthal RE. Oximetry-guided reoxygenation improves neurological outcome after experimental cardiac arrest. Stroke 2006;37:3008-13.

4. Hazelton JL, Balan I, Elmer Gl, et al. Hyperoxic reperfusion after global cerebral ischemia promotes inflammation and long-term hippocampal neuronal death. J Neurotrauma 2010;27:753-62.

5. Henlin T, Michalek P, Tyll T, Hinds JD, Dobias M. Oxygenation, ventilation, and airway management in out-of-hospital cardiac arrest: a review. Biomed Res Int 2014;2014:376871.

6. Liu Y, Rosenthal RE, Haywood Y, Miljkovic-Lolic M, Vanderhoek JY, Fiskum G. Normoxic ventilation after cardiac arrest reduces oxidation of brain lipids and improves neurological outcome. Stroke 1998;29:1679-86.

7. Vereczki V, Martin E, Rosenthal RE, Hof PR, Hoffman GE, Fiskum G. Normoxic resuscitation after cardiac arrest protects against hippocampal oxidative stress, metabolic dysfunction, and neuronal death. J Cereb Blood Flow Metab 2006;26:821-35.

8. Zwemer CF, Whitesall SE, D'Alecy LG. Cardiopulmonary-cerebral resuscitation with 100\% oxygen exacerbates neurological dysfunction following nine minutes of normothermic cardiac arrest in dogs. Resuscitation 1994;27:159-70.

9. Kilgannon $J H_{\text {, Jones }} A E_{\text {, Parrillo } J E}$, et al. Relationship between supranormal oxygen tension and outcome after resuscitation from cardiac arrest. Circulation 2011;123:2717-22.

10. Kilgannon JH, Jones $A E$, Shapiro $N I$, et al. Association between arterial hyperoxia following resuscitation from cardiac arrest and in-hospital mortality. JAMA 2010;303:2165-71.

11. Ferguson RP, Phelan T, Haddad T, Hinduja A, Dubin NH. Survival after in-hospital cardiopulmonary resuscitation. South Med J 2008;101:1007-11.

12. Ishtiaq $\mathrm{O}$, Iqbal $M$, Zubair $M$, Qayyum $R$, Adil M. Outcome of cardiopulmonary resuscitation: predictors of survival. J Coll Physicians Surg Pak 2008;18:3-7.

13. Khan NU, Razzak JA, Ahmed H, et al. Cardiopulmonary resuscitation: outcome and its predictors among hospitalized adult patients in Pakistan. Int J Emerg Med 2008;1:27-34.

14. Nadkarni VM, Larkin GL, Peberdy MA, et al. First documented rhythm and clinical outcome from in-hospital cardiac arrest among children and adults. JAMA 2006;295:50-7.

15. Bellomo R, Bailey M, Eastwood GM, et al. Arterial hyperoxia and in-hospital mortality after resuscitation from cardiac arrest. Crit Care 2011;15:R90.

16. Kuisma M, Boyd J, Voipio V, Alaspaa A, Roine RO, Rosenberg P. Comparison of 30 and the 100\% inspired oxygen concentrations during early post-resuscitation period: a randomised controlled pilot study. Resuscitation 2006;69:199-206.

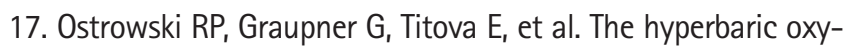
gen preconditioning-induced brain protection is mediated by a reduction of early apoptosis after transient global cerebral ischemia. Neurobiol Dis 2008;29:1-13.

18. Rosenthal RE, Silbergleit R, Hof PR, Haywood Y, Fiskum G. Hyperbaric oxygen reduces neuronal death and improves neurological outcome after canine cardiac arrest. Stroke 2003;34: 1311-6.

19. Takahashi M, Iwatsuki N, Ono K, Tajima T, Akama M, Koga Y. Hyperbaric oxygen therapy accelerates neurologic recovery after 15-minute complete global cerebral ischemia in dogs. Crit Care Med 1992;20:1588-94.

20. Jacobs I, Nadkarni V, Bahr J, et al. Cardiac arrest and cardiopulmonary resuscitation outcome reports: update and simplification of the Utstein templates for resuscitation registries: a statement for healthcare professionals from a task force of the international liaison committee on resuscitation (American Heart Association, European Resuscitation Council, Australian Resuscitation Council, New Zealand Resuscitation Council, Heart and Stroke Foundation of Canada, InterAmerican Heart Foundation, Resuscitation Council of Southern Africa). Resuscitation 2004;63:233-49.

21. Langhelle A, Nolan J, Herlitz J, et al. Recommended guidelines for reviewing, reporting, and conducting research on post-resuscitation care: the Utstein style. Resuscitation 2005;66:271-83.

22. Douzinas EE, Patsouris E, Kypriades EM, et al. Hypoxaemic reperfusion ameliorates the histopathological changes in the pig brain after a severe global cerebral ischaemic insult. Intensive Care Med 2001;27:905-10.

23. Abraham E, Matthay MA, Dinarello CA, et al. Consensus conference definitions for sepsis, septic shock, acute lung injury, and acute respiratory distress syndrome: time for a reevaluation. Crit Care Med 2000;28:232-5.

24. Bloom HL, Shukrullah I, Cuellar JR, Lloyd MS, Dudley SC Jr, Zafari AM. Long-term survival after successful inhospital cardiac arrest resuscitation. Am Heart J 2007;153:831-6. 
25. Solevag AL, Dannevig I, Nakstad B, Saugstad OD. Resuscitation of severely asphyctic newborn pigs with cardiac arrest byusing $21 \%$ or 100\% oxygen. Neonatology 2010;98:64-72.

26. Haynes RL, Baud O, Li J, Kinney HC, Volpe JJ, Folkerth DR. Oxidative and nitrative injury in periventricular leukomalacia: a review. Brain Pathol 2005;15:225-33.

27. Bayir H, Kagan VE, Clark RS, et al. Neuronal NOS-mediated nitration and inactivation of manganese superoxide dismutase in brain after experimental and human brain injury. J Neurochem 2007;101:168-81.

28. Martin E, Rosenthal RE, Fiskum G. Pyruvate dehydrogenase complex: metabolic link to ischemic brain injury and target of oxidative stress. J Neurosci Res 2005;79:240-7.
29. Wang $\mathrm{CH}$, Chang WT, Huang $\mathrm{CH}$, et al. The effect of hyperoxia on survival following adult cardiac arrest: a systematic review and meta-analysis of observational studies. Resuscitation 2014;85:1142-8.

30. Chen YS, Chao A, Yu HY, et al. Analysis and results of prolonged resuscitation in cardiac arrest patients rescued by extracorporeal membrane oxygenation. J Am Coll Cardiol 2003; 41:197-203.

31. Chen YS, Lin JW, Yu HY, et al. Cardiopulmonary resuscitation with assisted extracorporeal life-support versus conventional cardiopulmonary resuscitation in adults with in-hospital cardiac arrest: an observational study and propensity analysis. Lancet 2008;372:554-61. 